\title{
Système de transmission numérique d'images fixes réalisé à partir d'appareils de mesure
}

\author{
Hervé Boeglen ${ }^{l}$, Christian Chatellier ${ }^{2}$ et Olivier Haeberle $^{l}$ \\ ${ }^{1}$ Laboratoroire MIPS, IUT Mulhouse, 61 rue Albert Camus, 68093 Mulhouse Cedex France \\ ${ }^{2}$ Laboratoire SIC, Téléport 2, Bd Marie et Pierre Curie, 86962 Futuroscope Cedex France \\ herve.boeglen@uha.fr, chatellierdsic.sp2mi.univ-poitiers.fr, olivier.haeberle@uha.fr
}

\begin{abstract}
Résumé
Dans cet article, nous décrivons la mise en auvre d'un système de transmission numérique d'images compressées pratique réalisé à partir d'appareils de mesure. Deux appareils classiques (générateur vectoriel et analyseur) ont été détournés de leur but premier et ont été reliés via un bus au standard VISA à deux ordinateurs assurant, l'un les fonctions d'émission (compression, codage, modulation, filtrage des impulsions), l'autre de réception (CAG, synchronisation, démodulation, décodage, décompression) en bande de base du système de transmission numérique. La liaison $H F$ est réalisée à une fréquence de $2,4 \mathrm{GHz}$ et utilise des antennes WIFI " homemade » de type " cantenna ». Ce système, présenté à la Fête de la Science 2006, est une plateforme qui permet de mettre en ouvre et de valider des algorithmes de transmission entièrement numériques habituellement implémentés à l'aide de DSP et FPGA dans les systèmes de communication numériques modernes.
\end{abstract}

\section{Mots clefs \\ Communications numériques, compression d'images, synchronisation}

\section{Introduction}

Avec l'avènement des DSP et autres FPGA, la part de l'analogique dans les systèmes de communication ne cesse de diminuer et se retrouve bien souvent cantonnée à la translation de spectre et l'amplification HF de puissance. Cette évolution technologique impose une connaissance de la mise en œuvre d'algorithmes de traitement numérique du signal dont la méthodologie de conception requiert des compétences certaines en informatique. D'autre part, les instruments de mesure permettant d'évaluer les performances de tels systèmes ont également suivi cette évolution technologique puisqu'ils intègrent pour la plupart, un système d'exploitation moderne et des possibilités de communication très évoluées.

L'opportunité de disposer de tels instruments ainsi que la nécessité d'évaluer expérimentalement un système de compression d'images fixes robuste en transmission développé au laboratoire, nous a conduit à élaborer le système de communication numérique pratique que nous présentons ci-après.

Dans une première partie nous décrivons l'environnement matériel et logiciel du système ainsi que ses possibilités. La deuxième partie sera consacrée à la description de quelques algorithmes clés. Nous ferons état des résultats obtenus dans une troisième partie avant de conclure sur les utilisations et les évolutions possibles du système.

\section{D'un système de mesure à un système de transmission}

La Figure 1 ci-dessous présente le synoptique du système :

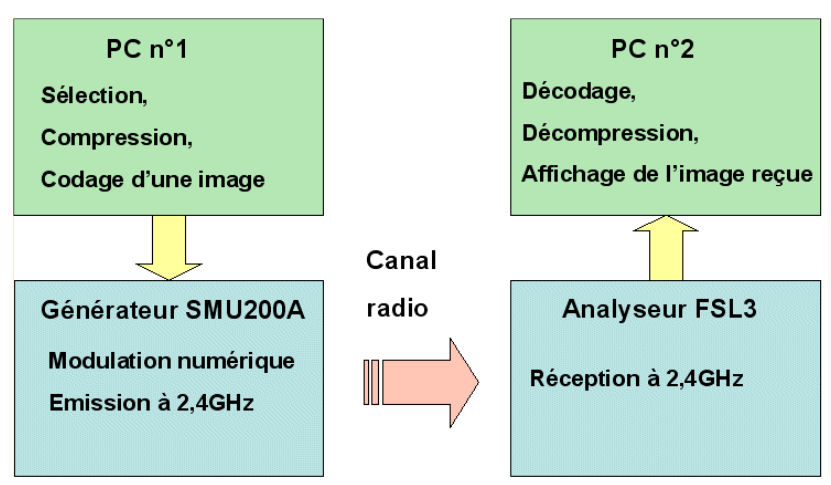

Figure 1 : Synoptique du système de transmission

Le système de mesure est composé d'un générateur vectoriel (émetteur) et d'un analyseur de spectre (récepteur) dont nous allons maintenant décrire les caractéristiques principales.

\subsection{Emission : le générateur vectoriel SMU200A}

Il s'agit d'un générateur vectoriel haut de gamme de la société Rohde \& Schwarz dont la profondeur de modulation IQ peut atteindre $200 \mathrm{MHz}$. La fréquence maximale du générateur RF est, pour la version que nous utilisons, de $3 \mathrm{GHz}$. Ce type d'appareil peut fonctionner selon deux modes principaux :

- $\quad$ Mode autonome :

Dans ce mode, l'appareil génère lui-même la séquence de bits à transmettre en utilisant son 
générateur pseudo-aléatoire interne (Pseudo Random Bit Sequence de 9 à 23 bits). Après un filtrage de Nyquist, les impulsions obtenues génèrent une modulation numérique sur fréquence porteuse (les modulations numériques classiques sont implémentées en standard)

- Mode arbitraire :

Dans ce mode, l'appareil lit les données I et Q depuis sa mémoire interne (64Méch dans notre cas) préalablement chargée par l'utilisateur. Ce mode permet de réaliser des transmissions à l'aide de données réelles (images, son etc.).

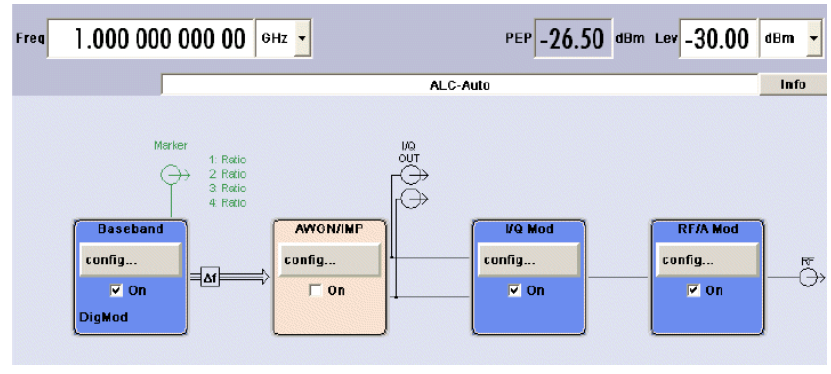

Figure 2 : Vue de l'écran de l'appareil

Comme notre application consiste à transmettre des images, nous utilisons l'appareil en mode arbitraire. Le rôle du PC connecté à l'appareil va donc consister à générer les vecteurs $\mathrm{I}$ et $\mathrm{Q}$ correspondants à l'image à transmettre.

L'appareil intègre un système d'exploitation de type Windows embarqué et permet de générer tous les standards de communication modernes (GSM, UMTS, DVB etc.). L'ensemble des fonctionnalités de l'instrument est accessible depuis sa face avant où en mode distant en passant par un bus de mesure. La Figure 2 montre une vue de l'écran de l'appareil où l'on voit clairement représentés les blocs de la chaîne de transmission mise en œuvre et notamment un générateur de bruit blanc Gaussien (bloc orange). On pourra se référer au manuel du SMU200A pour de plus amples détails sur ses fonctionnalités [1].

\subsection{Réception : l'analyseur de spectre FSL3}

Il s'agit d'un analyseur de spectre d'entrée de gamme de la société Rohde \& Schwarz qui présente l'avantage d'intégrer un démodulateur IQ ayant une capacité de 512kéch (Cf. Figure 3.).

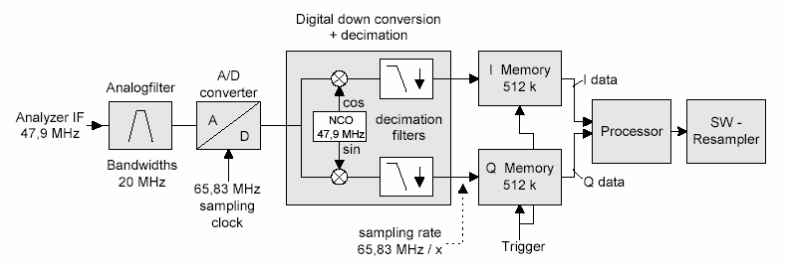

Figure 3 : Démodulateur IQ du FSL3
Il permet de travailler jusqu'à une profondeur de modulation de $20 \mathrm{MHz}$. La fréquence d'échantillonnage est paramétrable et le déclenchement de l'acquisition peut se faire sur un évènement extérieur ou sur un niveau de puissance de l'amplificateur de fréquence intermédiaire (bursts de synchronisation).

Comme le SMU200A, cet appareil intègre un système d'exploitation de type Windows embarqué et ses fonctionnalités sont accessibles via un bus de mesure. Pour une description détaillée de ses caractéristiques on pourra se référer à son manuel technique [1].

Ces deux appareils présentent l'avantage de disposer d'une interface LAN qui permet d'envisager un transfert beaucoup plus rapide des données que par le traditionnel bus GPIB. On peut, grâce à cette interface les piloter facilement à partir d'une couche logicielle de type VISA que nous décrivons dans le prochain paragraphe.

\subsection{L'environnement logiciel}

$\underline{\text { La communication avec les appareils }}$

L'environnement logiciel permettant la communication avec les appareils est décrit sur la Figure 4. ci-dessous :
PC

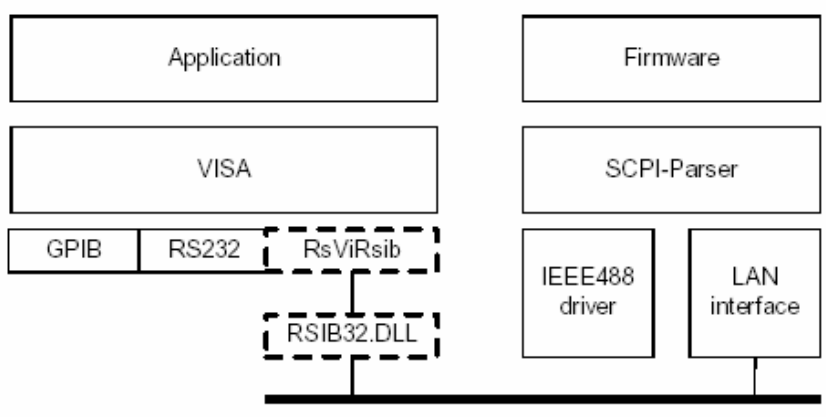

LAN
NI-VISA est une API logicielle qui réduit considérablement le temps de développement des systèmes de test et de mesure. Elle donne la possibilité aux développeurs de communiquer avec n'importe quel instrument, sur n'importe quel bus, et sur la plupart des systèmes d'exploitation utilisés aujourd'hui. Elle permet aussi la génération de code qui peut être porté d'une plateforme à une autre ou d'un bus à un autre avec peu ou pas de frais en terme de modification. Pour pouvoir l'utiliser avec l'interface LAN de nos appareils, la société Rohde \& Schwarz a développé une couche logicielle appelée RSIB Passport for VISA qui fait le lien entre le format des commandes GPIB des appareils et la syntaxe VISA.

Une fois les appareils connectés en réseau et les «briques » logicielles installées, on peut communiquer facilement avec eux depuis n'importe quelle application Windows à l'aide de commandes très simples comme en témoignent les lignes de code suivantes permettant de 
récupérer des vecteurs IQ provenant du FSL03 piloté depuis Matlab :

Ressource RSIB VISA sur adresse 192.168.1.244 vr = visa('ni','RSIB::192.168.1.244::INSTR'); oTaille du buffer de transfert en octets pour les données

set (vr, 'InputBufferSize', 2200000)

o Ouverture de la communication avec le FSL

fopen (vr);

-Activation du sous-système IO de l'instrument

fprintf(vr, 'TRAC:IQ:STAT ON');

oFiltre de résolution $=300 \mathrm{~Hz}$, fe du démodulateur $I Q=6 \mathrm{MHz}$

oSynchro ExTerne, front PoSitif, buffer de 0 à 269964 (sur 512k)

fprintf(vr,

TRAC : IQ : SET

NORM, $300 \mathrm{~Hz}, 6 \mathrm{MHz}, \mathrm{EXT}, \mathrm{POS}, 0,269964^{\prime}$ );

On lance l'initialisation de l'instrument et on

attend la fin

fprintf(vr, 'INIT; *WAI');

\% On fixe le format des données à récupérer

fprintf(vr, 'FORMAT REAL, 32');

on lance une acquisition

fprintf(vr, 'TRAC:IQ:DATA?');

oLes deux premières châ̂nes de caractères forment

l'entête : \# + longueur

ode la chaîne contenant la taille du buffer de

données en octets

header1 = fscanf (vr, 'ㅇ's', 2)

\%On récupère la taille des données en octets

header2 = fscanf(vr, '\%s', str2num(header1(2)))

\%Lecture des vecteurs I et 0

$\mathrm{xi}=$ fread (vr, str2num (header2)/8, 'float32').

$\mathrm{xq}=$ fread(vr, str2num(header2)/8,'float32');

oFin de la communication

fclose (vr)

Logiciels de développement des algorithmes bande de $\underline{\text { base }}$

Nous avons fait le choix du logiciel Matlab [2] car il permet le développement simple et rapide d'Interfaces Graphiques Utilisateur (IGU) comme le montre la Figure 5. ci-dessous.

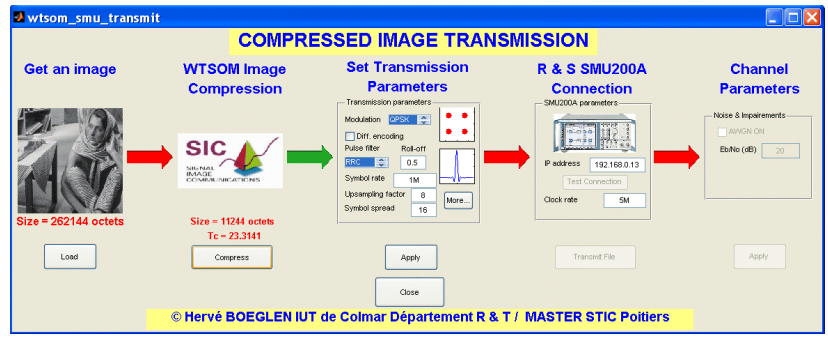

Figure 5 : IGU du PC émetteur

De plus, Matlab présente l'avantage de s'interfacer facilement avec des modules externes développés à l'aide de langages de haut niveau. Ainsi, certains algorithmes nécessitant un traitement rapide ont été implémentés en langage $\mathrm{C}++$ à partir de la librairie de communication numérique libre IT++ [3] puis compilés et intégrés à Matlab sous la forme de bibliothèques de lien dynamique (DLL).

\section{Détail des algorithmes mis en auvre}

Pour des raisons de temps de calcul, la simulation classique de systèmes de communications numérique n'intègre généralement pas les fonctions de synchronisation du rythme et de la phase ni de filtrage des impulsions (ce que les Anglo-Saxons appellent le «waveform channel »). La mise en œuvre d'un système réel ne peut évidemment pas faire l'économie de ces fonctions. C'est la raison pour laquelle nous allons nous focaliser sur la méthodologie et la mise œuvre de quelques algorithmes implémentant ces fonctionnalités.

\subsection{Algorithmes bande de base à l'émission}

Le générateur arbitraire du SMU200A accepte les vecteurs IQ de la modulation implémentée (QPSK, 8PSK ou 16QAM) au format virgule fixe Q15. Ces vecteurs sont générés dans Matlab à partir des bits de l'image à transmettre, sur-échantillonnés puis passés dans un filtre de Nyquist pour obtenir des impulsions temporelles comme on peut le voir sur la Figure 6.
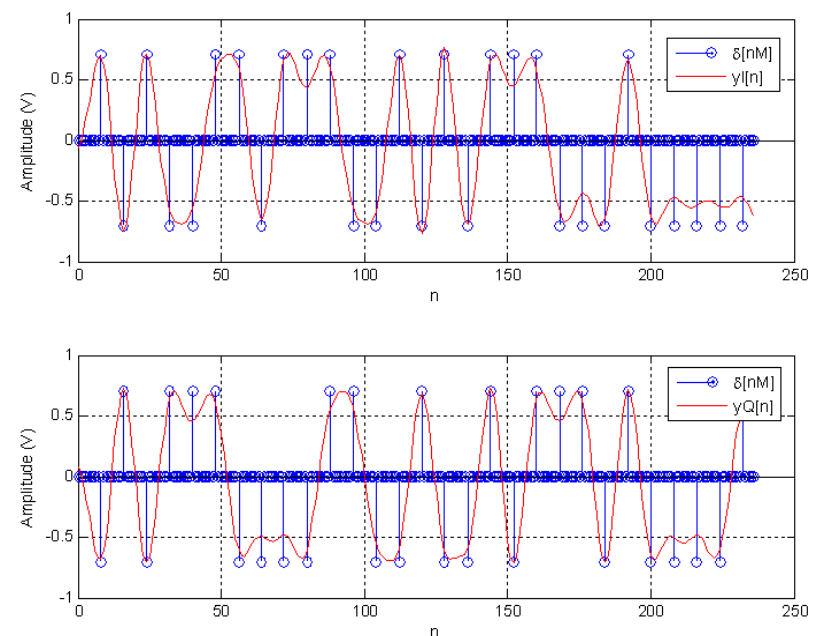

Figure 6 : Formes d'ondes I et $Q$ modulation $Q P S K$

Le schéma bloc suivant résume les opérations à réaliser pour obtenir les formes d'ondes de la Figure 6.

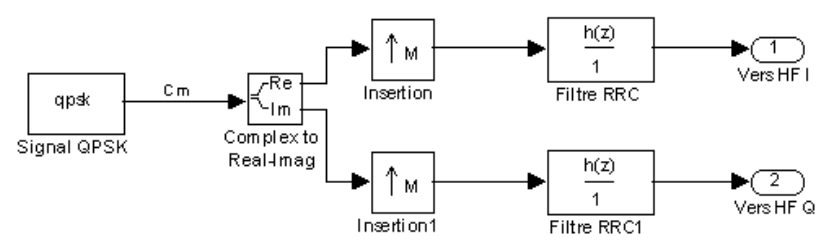

Figure 7 : Opérations à réaliser pour obtenir des impulsions temporelles

\subsection{Algorithmes bande de base à la réception}

La complexité du système se concentre au niveau du récepteur, car en plus des opérations inverses de celle de 
l'émetteur, il faut mettre en œuvre des algorithmes de Correction Automatique de Gain (CAG) de synchronisation du rythme et de la phase.

\section{$\underline{\mathrm{CAG}}$ :}

Celle-ci est rendue nécessaire par le fait que les étages de synchronisation sont conçus pour fonctionner à un niveau défini. Le schéma est conforme à la figure suivante.

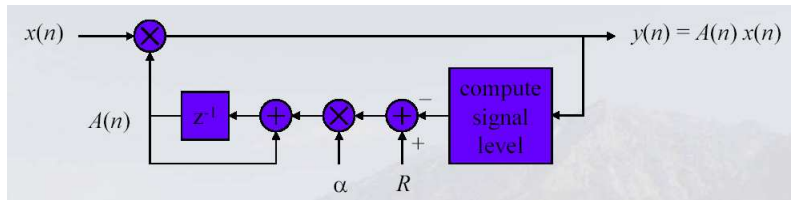

Figure 8:CAG numérique linéaire

Le sous-système de récupération du rythme :

En ce qui concerne la récupération du rythme, nous avons utilisé la possibilité qu'offre l'analyseur de spectre de se synchroniser sur un niveau de puissance. Ainsi, il suffit de configurer le générateur en mode «burst» de sorte qu'il émette une image selon un rapport cyclique prédéterminé. On s'assure alors qu'au niveau de la réception le démarrage de l'acquisition du démodulateur IQ se fasse sur le premier symbole de l'image. La figure suivante donne un exemple d'acquisition des vecteurs I et $\mathrm{Q}$.
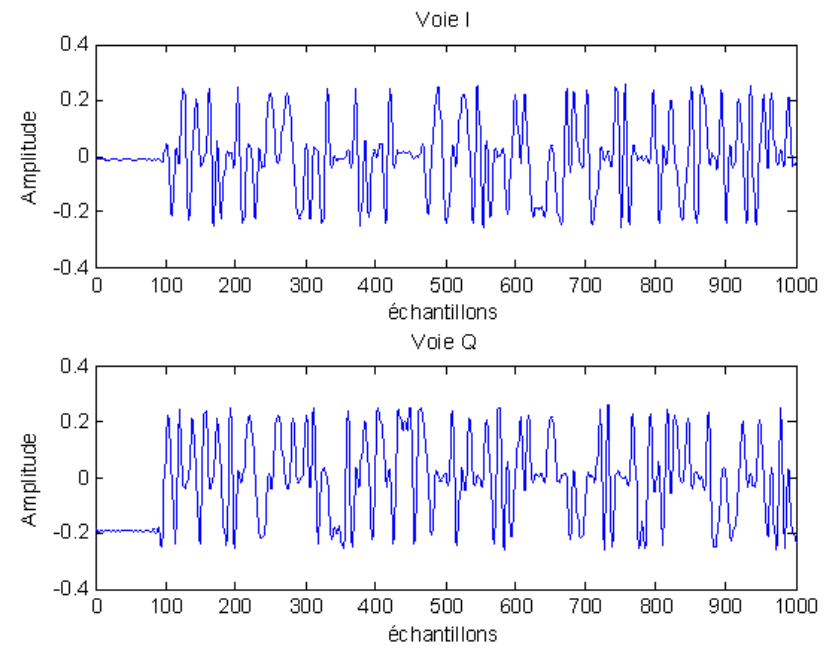

Figure 9 : Vecteurs I et $Q$ provenant du FSL3

Le sous-système de récupération de la phase :

La récupération de la phase utilise une structure du type boucle de Costas à retour de décision [4][5]. Sa structure est conforme à la Figure 10 pour la modulation QPSK.

Le bloc de décision de la Figure 10. fonctionne selon le critère de la minimisation de la distance Euclidienne entre les symboles théoriques et les symboles reçus et dépend du type de modulation utilisé. La Figure 11 donne un exemple de constellation 16QAM avant et après correction de la phase.

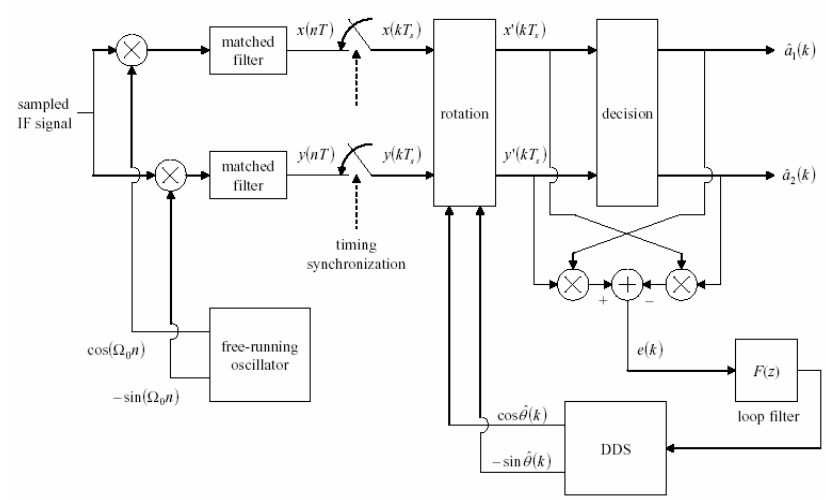

Figure 10 : Boucle de récupération de phase

Suivant le type de modulation utilisée, la courbe en $\mathrm{S}$ du détecteur de phase possède plusieurs points stables de verrouillage. Cela conduit à une ambiguïté de phase que l'on peut lever selon deux techniques classiques [6] :

- encodage différentiel des données

- utilisation de la technique du mot unique (Unique Word) qui consiste à placer une séquence connue (pilotes) parmi les données. En fonction de la valeur de cette séquence à la réception on pourra déterminer la valeur de l'ambiguïté de phase puis corriger l'ensemble des données.
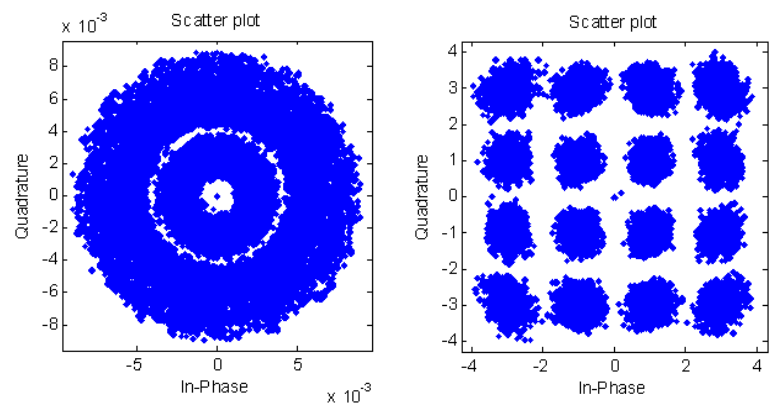

Figure 11 : Constellation 16QAM avant et après correction de phase

La démarche de conception de ce sous-système a dans un premier temps, consisté à convertir les équations d'une Boucle à Verrouillage de Phase (BVP) analogique du $2^{\text {ème }}$ ordre en son équivalent numérique [7]. Dans un deuxième temps, la structure conforme à la Figure 10 a été implémentée pour validation dans Simulink [2]. Enfin, le modèle Simulink a été converti en un module écrit en langage $\mathrm{C}++$ puis compilé et intégré à Matlab. Ce module traite 40000 symboles en $0,1 \mathrm{~s}$. Il nécessite toutefois d'ajouter aux données un entête de 20 symboles pour tenir compte du temps de verrouillage de la BVP.

Mise en œuvre d'un égaliseur de type Recursive Least Squares (RLS) :

Ce type d'égaliseur a été choisi pour sa rapidité de convergence. Le fonctionnement se fait selon deux modes successifs :

- apprentissage,

- retour de décision 
La mise en œuvre d'une séquence d'apprentissage nécessite d'ajouter aux données un entête connu du récepteur et dont la longueur doit être supérieure au temps de convergence de l'algorithme. Selon la modulation et les caractéristiques du canal, des longueurs variant de 20 à 100 symboles ont été nécessaires.

Même si le temps de traitement est triple de celui de la méthode précédente, l'égaliseur est plus robuste et présente l'avantage de corriger l'IES, conséquence de la sélectivité fréquentielle du canal. De plus, il permet d'obtenir la réponse impulsionnelle de ce dernier. En outre, l'opération d'égalisation intègre de manière sousjacente les opérations de $\mathrm{CAG}$, de récupération du rythme et de la phase.

La Figure 12. donne un exemple de constellation après égalisation ainsi que l'évolution de l'Erreur Quadratique Moyenne (EQM) au cours du temps.
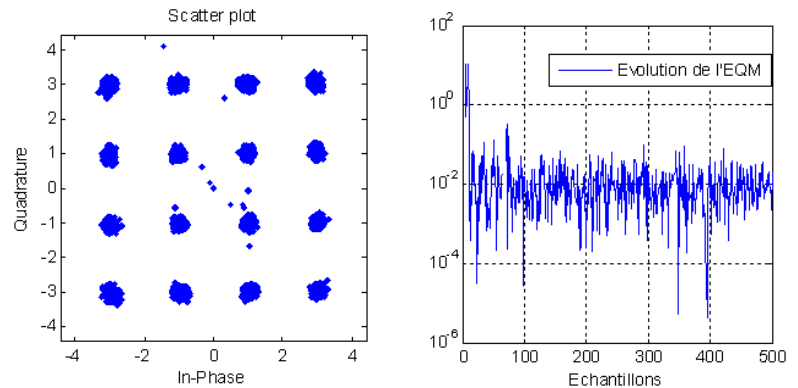

Figure 12 : Constellation 16QAM après égalisation, évolution de l'EQM de l'égaliseur

\section{Résultats}

Les différents algorithmes décrits précédemment ont été testés avec succès en établissant une liaison sans fil à l'aide de deux antennes WIFI de type «cantenna » sur des distances allant de 10 à 50 mètres pour une puissance d'émission de $0 \mathrm{dBm}$.

Ce système de transmission a également permis la validation d'un procédé de compression d'images fixes appelé WTSOM (Wavelet Transform Self-Organizing Map) [8] particulièrement robuste aux erreurs de transmission puisqu'il permet d'obtenir des images de bonne qualité visuelle jusqu'à un de Taux d'Erreur Binaire (TEB) de 3\%. La Figure 15. donne un exemple de transmission de ce type d'image.

Les performances du système pratique ont été comparées aux performances obtenues en simulation. On constate une perte comprise entre 2,5 et $4 \mathrm{~dB}$ suivant le type de modulation utilisée dans une configuration de type canal Gaussien. Toutefois, même lorsque les deux antennes sont en lien direct sans obstacle, nous avons constaté la présence de multitrajets conduisant à une faible sélectivité fréquentielle du canal. Ce canal ne peut donc pas être considéré comme simplement Gaussien !

\section{Conclusion}

Nous avons présenté un système de communication numérique réalisé à partir d'appareils de mesure. Ce système a permis, d'une part de valider des algorithmes de synchronisation et d'égalisation et d'autre part de vérifier expérimentalement la robustesse d'un procédé de compression d'images fixes développé au laboratoire.

Nous avons également décidé de valoriser ce travail dans la direction du grand public en présentant la manipulation à l'édition 2006 de la Fête de la Science. Les étudiants de $2^{\text {ème }}$ année de DUT Réseaux et Télécommunication (R\&T) chargés de l'animation du stand ont montré beaucoup d'intérêt à la manipulation. Ils prenaient les personnes volontaires avec un appareil photo numérique, puis après transfert de la photo sur le PC d'émission, ils effectuaient la transmission de l'image tout en expliquant les rudiments de la compression et de la transmission numérique. L'enthousiasme manifesté par les étudiants lors de cette manifestation nous a amené à utiliser le système pour illustrer les cours de transmission numérique de $2^{\text {ème }}$ année de DUT R\&T (Colmar) et de Master STIC (Poitiers)

Enfin, l'environnement logiciel utilisé permet d'envisager une évolution simple et rapide du système. Nous projetons notamment l'implémentation de la modulation OFDM [9] connue pour ses bonnes performances sur des canaux sélectifs en fréquence.

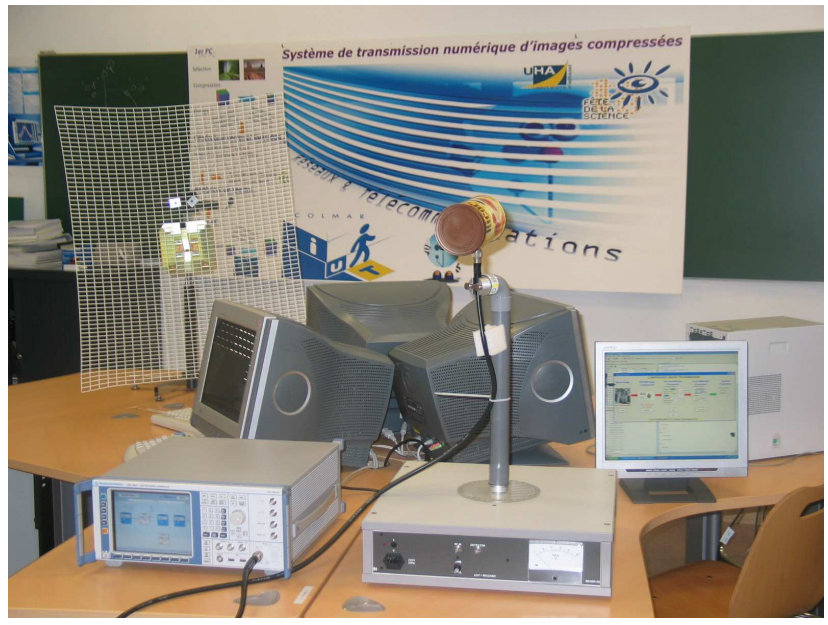

Figure 13 : Partie émission

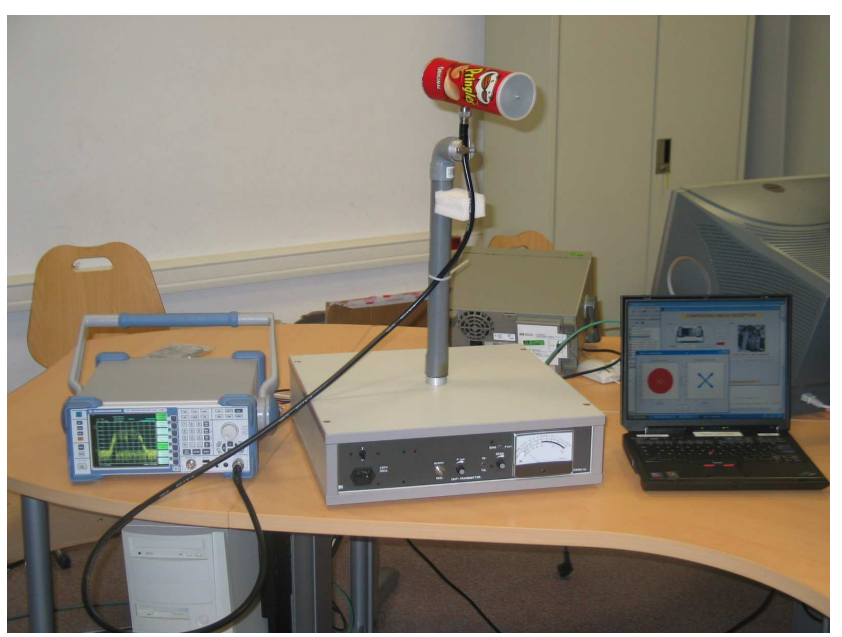

Figure 14 : Partie réception 


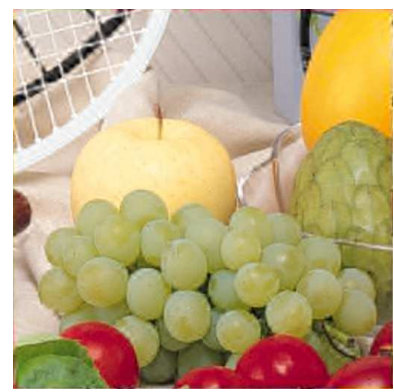

a) image WTSOM transmise $\mathrm{PSNR}=26.33 \mathrm{~dB}$

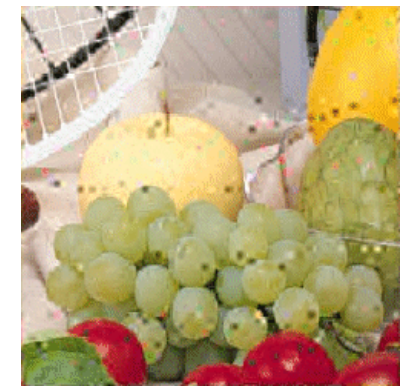

b) image WTSOM reçue, après correction d'erreurs $\mathrm{TEB}=1.6 \%, \mathrm{PSNR}=24.2 \mathrm{~dB}$

Figure.15. : Transmission WTSOM QPSK SNR=6dB

\section{Références}

[1] http://www.rohde-schwarz.com

[2] http://www.mathworks.com

[3] http://itpp.sourceforge.net/

[4] C. Chatellier, J. Brochard, Récupération de porteuse dans les systèmes de transmissions analogiques et numériques, J3eA, Vol. 3 (2004) 5.

[5] U. Mengali, A. D'Andrea. Synchronization techniques for digital receivers. Plenum Press, 1997.

[6] M. Rice Synchronization notes, Brigham Young University, 2001.

[7] W. Lindsey, A survey of digital phase-locked loops, Proceedings of the IEEE, Vol. 69, No. 4, April 1981

[8] C. Chatellier, P. Bourdon, B. Souhard, C. Olivier, "An efficient joint source channel coding scheme for image transmission through the ionospheric channel", European Conference Propagation and Systems, Brest France, March 2005.

[9] J.A.C. Bingham, Multicarrier modulation for data transmission: an idea whose time has come, IEEE Communications Magazine, Volume 28, Issue 5, May 1990 Page(s):5 - 14 